hep-th/0402135

SLAC-PUB-10347

SU-ITP-04/06

\title{
The Scaling of the No-Scale Potential and de Sitter Model Building
}

\begin{abstract}
Alex Saltman and Eva Silverstein *
We propose a variant of the KKLT (A)dS flux vacuum construction which does not require an antibrane to source the volume modulus. The strategy is to find nonzero local minima of the no-scale potential in the complex structure and dilaton directions in moduli space. The corresponding no-scale potential expanded about this point sources the volume modulus in the same way as does the antibrane of the KKLT construction. We exhibit explicit examples of such nonzero local minima of the no-scale potential in a simple toroidal orientifold model.
\end{abstract}

\footnotetext{
* SLAC and Department of Physics, Stanford University, Stanford, CA 94309
} 


\section{Introduction}

De Sitter and anti-de Sitter vacua of string theory that fix all moduli were constructed recently [1,2,3]. The de Sitter models offer the prospect of modelling dark energy and inflation in string theory, as well as providing a concrete setting in which to analyze basic quantum gravitational issues (such as the de Sitter entropy [4,5] and the rich cosmology and particle physics of multiple vacua [6,0,8]).

The KKLT models [2] may be of particular interest as they arise in a context with low-energy supersymmetric effective field theory and naturally incorporate other appealing model-building features, such as warping to obtain small numbers. In this paper we will provide explicit examples of a simple variant of the KKLT construction.

One ingredient of the model [2] is an anti-D3-brane in a warped 3-brane throat of a Calabi-Yau compactification of type IIB string theory [9]. This produces a contribution to the four-dimensional Einstein-frame potential for the overall volume $V_{C Y}$ of the Calabi-Yau

$$
V_{\overline{D 3}} \sim \frac{1}{V_{C Y}^{2}} \tilde{V}
$$

where $\tilde{V}$ does not depend on the overall volume, to a first approximation, and is tunably small if the $\overline{D 3}$ sits at the bottom of a sufficiently warped throat. The contribution (1.1) can be understood simply as arising from the $\overline{D 3}$ tension in four-dimensional Einstein frame. It is not yet precisely known how to package the contributions of the $\overline{D 3}$-brane in terms of the superpotential, Kähler potential, and D terms of a low energy supersymmetric effective field theory. (Recently, an interesting variant of KKLT has been proposed in which the antibrane is embedded in a 7-brane, giving a D-term realization of it [10]; it will be interesting to see if explicit tadpole and tachyon free models arise also in that context.)

The potential energy of a generic model can be written in $\mathcal{N}=1$ supersymmetric form in terms of a Kähler potential $K$ and superpotential $W$ as

$$
V=e^{K}\left(\sum_{a, b} g^{a \bar{b}} D_{a} W \overline{D_{b} W}-3|W|^{2}\right)+\mathrm{D} \text { terms }
$$

where $a$ and $b$ run over all moduli and the axion-dilaton and $g_{a \bar{b}}=\partial_{a} \partial_{\bar{b}} K$ is the metric on moduli space. In a no-scale model, such as those developed in [11], this reduces to

$$
V_{\text {no-scale }}=e^{K}\left(\sum_{i, j} g^{i \bar{j}} D_{i} W \overline{D_{j} W}\right)
$$


where $i$ and $j$ run over only complex structure moduli and the axion-dilaton (collectively $\left.\Phi_{i}\right)$, the Kähler and D3-brane position moduli having cancelled out. Thus far, solutions to this potential have been found [2, 11, [12, 13, [1], by solving $D_{i} W=0, \forall i$, which produces a zero-energy minimum of (1.3) with fixed complex structure moduli. The Kähler moduli only appear in (1.3) in the prefactor $e^{K}$, so they are fixed by other ingredients-a balance of forces from (1.1) and two terms arising from no-scale breaking effects ( $\alpha^{\prime}$ corrections and nonperturbative effects). This leaves the complex moduli close to their original positions as stabilized by the no-scale potential.

However, the functional form of (1.3) immediately suggests an interesting alternative to this construction. The overall volume appears as a Kähler modulus in a chiral multiplet $\rho$ whose imaginary part is $V_{C Y}^{2 / 3} / g_{s}$, and $e^{K} \propto 1 /(\operatorname{Im} \rho)^{3} \propto 1 / V_{C Y}^{2}$. So if there is a minimum of the potential in the complex-structure and axion-dilaton directions at nonzero $V_{\text {no-scale, }}$

$$
\left.\partial_{i} V\right|_{\Phi_{i}^{*}}=0 \quad V\left(\Phi_{i}^{*}\right)>0
$$

with the eigenvalues of $\partial_{i} \partial_{j} V$ positive, it provides a source for $\rho$ which is identical in form to that provided by the antibrane (1.1).

In a similar manner to [2], we can play this positive-power-law potential (1.1) against contributions coming from no-scale-violating effects. For example, it is straightforward to expand the potential (1.2) by taking $W \rightarrow W_{n s}+\Delta W$ where $\Delta W$ is non-perturbatively small in the volume modulus and $W_{n s}$ is the no-scale superpotential (independent of the volume). Combining the resulting terms with our positive potential, we obtain contributions scaling like $\sqrt{\tilde{V}} \Delta W /(\operatorname{Im} \rho)^{3}$ and $\Delta W /(\operatorname{Im} \rho)^{4}$ along with the $\tilde{V} /(\operatorname{Im} \rho)^{3}$ from (1.1); with appropriate signs, if $\tilde{V}$ is small, these terms can play off each other to fix the volume at a large value. In the example of [2], the small number required is obtained by positioning the antibrane at the bottom of a warped throat. In our case, we will have to rely on the richness of the set of possible fluxes to obtain a small number.

This class of models is in some sense a natural completion of that offered by KKLT, since there are domain walls coming from wrapped fivebranes on which the antibrane (as well as other threebranes) can end. So the set of models without an antibrane is physically connected to the original set of KKLT models through bubble nucleation [15, 6, 16], and via the deformation of the system onto its approximate Coulomb branch [5, 团].

Our strategy has the advantage that the supersymmetric effective field theory description is clear, and shows that there is no a priori need for warped throats. In realizing our 
idea with explicit examples, we will work with toroidal orientifolds as in 17, 12,13, rather than using generic warped Calabi-Yau manifolds. As in the early work on string compactification phenomenology, there is a practical trade-off between genericity and calculability. We will also find it very useful to work near a locus of enhanced symmetry within the toroidal orientifold model's moduli space. Again this entails some loss of generality, but on the other hand there are various interesting physical mechanisms and scenarios which favor symmetries (such as [18,19] and avoidance of proton decay).

\section{A Concrete Model: De-Sitter Vacua of the $T^{6} / \mathbb{Z}_{2}$ Orientifold}

In the KKLT models, before $\alpha^{\prime}$ and nonperturbative correction, the potential takes the form (1.3), with Kähler potential

$$
K=-3 \ln [-i(\rho-\bar{\rho})]-\ln [-i(\phi-\bar{\phi})]-\ln \left[-i \int_{M} \Omega \wedge \bar{\Omega}\right]
$$

and superpotential [20,21,22]

$$
W=\int_{M} G_{3} \wedge \Omega
$$

Here $\phi$ is the IIB axiodilaton (a departure from the notation of [2] ) and $\rho=b / \sqrt{2}+$ $i(\operatorname{Im} \phi) e^{4 u}$, where $b$ is the integral of the NS 2-form potential over the 2-cycle corresponding to the overall volume Kähler modulus and $e^{u}$ scales with the linear size of the Calabi-Yau.

Thus the $\rho$ dependence of the potential is simply a multiplicative factor of $1 /(\operatorname{Im} \rho)^{3}$ from the Kähler potential as mentioned in $\S 1$.

The potentials (1.3) that one obtains from generic Calabi-Yau manifolds have a rich structure, and though the moduli spaces are of high dimension, we expect them to produce metastable solutions of (1.4). But, for specificity, we now specialize to the case of flux compactifications of type IIB on the $T^{6} / \mathbb{Z}_{2}$ orientifold [12] [13.

\subsection{The $T^{6} / \mathbb{Z}_{2}$ geometry}

In fact this naively simpler case has several complications of its own coming from the fact that there is no preferred decomposition into Kähler and complex structure moduli. (This can be thought of in spacetime language as coming from the fact that the UV theory has $\mathcal{N}=4$ supersymmetry in four dimensions.) In particular, the derivation of the no-scale potential and flux superpotential (1.3) (2.2) breaks down in this case. Ultimately we will 
simply calculate the potential energy in components and minimize it, exhibiting explicit tachyon free models in a way which bypasses these subtleties.

In order to calculate potentials for moduli in various flux configurations, we introduce a convenient set of real global coordinates on $T^{6}: x^{i}, y^{i}, i=1, \ldots, 3$ with the identifications $x^{i} \equiv x^{i}+1, y^{i} \equiv y^{i}+1$. Choices of complex structure can be parameterized by complex numbers $\tau^{i j}, i, j=1, \ldots, 3$ such that

$$
z^{i}=x^{i}+\tau^{i j} y^{j}
$$

are global holomorphic coordinates. In these coordinates, the explicit orientifold is $T^{6} /\left(\Omega R(-1)^{F_{L}}\right)$ where $R:\left(x^{i}, y^{i}\right) \rightarrow-\left(x^{i}, y^{i}\right)$. The holomorphic three-form can be taken to be

$$
\Omega=d z^{1} \wedge d z^{2} \wedge d z^{3}
$$

and the metric can be chosen to be

$$
d s^{2}=d z^{i} d \bar{z}^{\bar{i}}
$$

In this parameterization, deformations of $\tau^{i j}$ induce deformations of the metric that are a combination of Kähler $\left(\delta g_{i \bar{j}} \neq 0\right)$ and complex structure $\left(\delta g_{i j} \neq 0\right)$ perturbations. In addition (independent of the parameterization), some deformations of $\tau^{i j}$ induce no complex-structure metric perturbation at all. We will call these unphysical deformations 2 .

It can be shown that the flux superpotential $(2.2)$ in the $T^{6} / \mathbb{Z}_{2}$ case [12] depends in general on all the $\tau^{i j}$, even those which are unphysical, evidence that the full supergravity description of this system [23] is more complicated than the naive extrapolation of (1.3) and (2.2) to the torus case. To avoid this complication, we turn to computing the quantities we will need from the dimensional reduction of the ten dimensional type IIB component Lagrangian.

1 It should be possible to correct the metric with some dependence on $\tau^{i j}$ that removes the Kähler perturbations, as is commonly done on $T^{2}$ by defining the Kähler form as $1 /(\bar{\tau}-\tau) d z d \bar{z}$. However, for simplicity, we will not make such a choice.

2 These exist because of the presence of non-trivial $(0,1)$-forms on the torus, and can be seen more directly by noting that $h^{2,1}=9$, but there are only 6 independent $\delta g_{i j}$ 


\subsection{Dimensional Reduction}

We follow [11] and [12] through most of this subsection. The Type IIB supergravity action in ten dimensional Einstein frame is [24]

$$
\begin{aligned}
S_{\text {IIB }} & =\frac{1}{2 \kappa_{10^{2}}} \int d^{10} x \sqrt{-g}\left(R-\frac{\partial_{M} \phi \partial^{M} \phi}{2(\operatorname{Im} \phi)^{2}}-\frac{G_{(3)} \cdot \bar{G}_{(3)}}{2 \cdot 3 ! \cdot \operatorname{Im} \phi}-\frac{\tilde{F}_{(5)}^{2}}{4 \cdot 5 !}\right) \\
& \left.+\frac{1}{2 \kappa_{10}{ }^{2}} \int \frac{C_{(4)} \wedge G_{(3)} \wedge \bar{G}_{(3)}}{4 i(\operatorname{Im} \phi}\right)+S_{\text {local }} .
\end{aligned}
$$

where

$$
\begin{aligned}
& \phi=C_{(0)}+i / g_{s}, \quad G_{(3)}=F_{(3)}-\phi H_{(3)}, \\
& \tilde{F}_{(5)}=F_{(5)}-\frac{1}{2} C_{(2)} \wedge H_{(3)}+\frac{1}{2} F_{(3)} \wedge B_{(2)},
\end{aligned}
$$

with $* \tilde{F}_{(5)}=\tilde{F}_{(5)}, F_{(3)}=d C_{(2)}$, and $H_{(3)}=d B_{(2)}$. If we compactify on a six dimensional compact manifold $\mathcal{M}_{6}$, the Bianchi identity for the 5 -form field strength is

$$
d \tilde{F}_{(5)}=d * \tilde{F}_{(5)}=H_{(3)} \wedge F_{(3)}+2 \kappa_{10}^{2} \mu_{3} \rho_{3}^{\text {local }}
$$

where $\rho_{3}^{\text {local }}$ is the number density of local D3-brane charge sources. This can be integrated over $\mathcal{M}_{6}$ to give

$$
\frac{1}{2 \kappa_{10} \mu_{3}} \int_{\mathcal{M}_{6}} H_{(3)} \wedge F_{(3)}+Q_{3}^{\text {local }}=0 .
$$

In the case of interest to us, the local sources of D3-brane charge are D3-branes, with charge +1 , and orientifold 3 -planes with charge $-1 / 4$. In the particular case of $T^{6} / \mathbb{Z}_{2}$, there are $2^{6}$ O3-planes, so the condition becomes

$$
\frac{1}{2} \frac{1}{(2 \pi)^{4}\left(\alpha^{\prime}\right)^{2}} \int_{T^{6}} H_{(3)} \wedge F_{(3)}+N_{\mathrm{D} 3}+\frac{1}{2} N_{\mathrm{O}^{\prime}}=16
$$

Since we wish to avoid $\overline{D 3}$-branes, this gives the constraint

$$
\frac{1}{(2 \pi)^{4}\left(\alpha^{\prime}\right)^{2}} \int_{T^{6}} H_{(3)} \wedge F_{(3)} \leq 32
$$

There are various types of exotic O3-planes that would contribute to (2.10), but we will impose constraints so that these do not appear in our constructions. In particular, we will use the quantization conditions

$$
\frac{1}{(2 \pi)^{2} \alpha^{\prime}} \int_{\gamma} F_{(3)}=m_{\gamma} \in 2 \mathbb{Z}, \quad \frac{1}{(2 \pi)^{2} \alpha^{\prime}} \int_{\gamma} H_{(3)}=n_{\gamma} \in 2 \mathbb{Z},
$$


so that $F_{(3)}$ and $H_{(3)}$ still obey the standard quantization conditions even though all three cycles are halved in volume by the $\mathbb{Z}_{2}$ action. This ensures that all of the O3-planes are of the standard type [13].

A convenient basis for $H^{3}\left(T^{6}\right)$ is

$$
\begin{aligned}
\alpha_{0} & =d x^{1} \wedge d x^{2} \wedge d x^{3} \\
\alpha_{i j} & =\frac{1}{2} \epsilon_{i l m} d x^{l} \wedge d x^{m} \wedge d y^{j}, \quad 1 \leq i, j \leq 3 \\
\beta^{i j} & =-\frac{1}{2} \epsilon_{j l m} d y^{l} \wedge d y^{m} \wedge d x^{i}, \quad 1 \leq i, j \leq 3 \\
\beta_{0} & =d y^{1} \wedge d y^{2} \wedge d y^{3}
\end{aligned}
$$

which satisfies

$$
\int_{\mathcal{M}_{6}} \alpha_{I} \wedge \beta^{J}=\delta_{I}^{J}
$$

For compactification on $T^{6}$, flux configurations of $F_{(3)}$ and $H_{(3)}$ take values in $H^{3}\left(T^{6}, \mathbb{Z}\right)$. As shown in [12], these configurations are all consistent with the orientifold action, with the quantization-condition caveat mentioned previously, so $F_{(3)}$ and $H_{(3)}$ can be generically expanded as

$$
\begin{aligned}
& \frac{1}{(2 \pi)^{2} \alpha^{\prime}} F_{(3)}=a^{0} \alpha_{0}+a^{i j} \alpha_{i j}+b_{i j} \beta^{i j}+b_{0} \beta^{0}, \\
& \frac{1}{(2 \pi)^{2} \alpha^{\prime}} H_{(3)}=c^{0} \alpha_{0}+c^{i j} \alpha_{i j}+d_{i j} \beta^{i j}+d_{0} \beta^{0}
\end{aligned}
$$

with $a^{0}, a^{i j}, b_{i j}, b_{0}, c^{0}, c^{i j}, d_{i j}, d_{0} \in 2 \mathbb{Z}$

For the reasons discussed in the previous subsection, we will study the component potential energy

$$
V_{\text {real }}=\frac{1}{24 \kappa_{10}^{2}(\operatorname{Im} \rho)^{3}} \int_{M} d^{6} y g^{1 / 2} \frac{G_{m n p} \bar{G}^{m n p}}{\operatorname{Im} \phi}-\frac{i}{4 \kappa_{10}^{2}(\operatorname{Im} \phi)(\operatorname{Im} \rho)^{3}} \int_{M} G_{(3)} \wedge \bar{G}_{(3)} \text {. }
$$

This comes from (2.5) after moving to four-dimensional Einstein frame, taking into account the fact that the second term is, by (2.10), the energy of the local sources.

\subsection{The symmetric locus}

In order to simplify our task, we will now specialize to an enhanced symmetry locus on the moduli space of the $T^{6}$. This allows us to first minimize the potential within the symmetric locus. Since the enhanced symmetry guarantees no tadpoles in the directions transverse to the symmetric locus, the potential is already extremized in those directions. 
What remains is then to study the mass matrix in the transverse directions to check for tachyons.

This simplification is crucial even for computer searches, which use a steepest descent method. The minima we for which we search are local minima with small "drainage basins." Generically the number of attempts required to find a minimum (even if we know it exists) will go as $\left(l^{\prime} / l\right)^{f}$ where $l$ is the scale of the basin, $l^{\prime}>l$ the characteristic distance of the basin from the origin, and $f$ the number of degrees of freedom. Furthermore, each attempt takes longer as $f$ increases, so minimizing $f$ is quite important. By implementing a symmetry constraint we reduce $f$ to the number of directions respecting the symmetry. Of course, we have no guarantee that the symmetry-breaking directions will be tachyon-free.

In our case, will impose the symmetry generated by $R_{1}:\left(x^{1}, x^{2}, x^{3}, y^{1}, y^{2}, y^{3}\right) \rightarrow$ $\left(-x^{1},-x^{2}, x^{3},-y^{1},-y^{2}, y^{3}\right)$ and $R_{2}:\left(x^{1}, x^{2}, x^{3}, y^{1}, y^{2}, y^{3}\right) \rightarrow\left(x^{1},-x^{2},-x^{3}, y^{1},-y^{2},-y^{3}\right)$. Under this action, only diagonal $\tau^{i j}$, the $\tau^{i i}$, are preserved, and the only invariant threeforms are $\alpha_{0}, \alpha_{i i}, \beta^{i i}$, and $\beta^{0}$, limiting the flux quantum numbers to $a^{0}, a^{i i}, b_{i i}$, and $b_{0}$. If we limit ourselves to complex structure moduli and the axion-dilaton, this leaves us with eight real degrees of freedom, representing the three diagonal tau and the axion-dilaton.

\subsection{Solutions}

Using the potential (2.16) restricted to the symmetric locus, with fixed $\rho$, we performed a randomized search for nonzero local minima with the "FindMinimum" function of Mathematica 4.1, which uses a modification of Powell's method for minimization [25]. We tried more than $10^{4}$ different relatively prime choices of flux quantum numbers $a, b, c, d \leq 10$, and for each flux choice, 50 attempts were made to find a nonzero minimum. We found four distinct, but possibly related, nonzero minima-their characteristics can be found in Appendix A3.

We then performed the second step (checking for tachyons in the transverse directions) by looking at the derivatives of the full potential (2.16) in terms of the full real metric and the axion-dilaton. All first derivatives were zero, which was expected for the symmetrybreaking Kähler directions and all complex structure directions. Interestingly, in all cases, almost all moduli were fixed, with only three or four massless directions remaining and no tachyons. These data are also in Appendix A.

3 One could, at this point, consider these to be solutions in the orientifold $T^{6} / \mathbb{Z}_{2}^{3}$ defined by the combined action. However, the flux quantization condition is not clear in this case, and under the most conservative choice, fluxes in $H^{3}\left(T^{6}, 8 \mathbb{Z}\right)$, the condition analogous to (2.11) is not satisfied by any of our solutions. 


\section{Conclusions}

We have exhibited tachyon-free models which satisfy (1.4). In order to stabilize the three or four remaining Kähler moduli, including the volume modulus, one would need to combine our contribution (1.1) with non-perturbative and $\alpha^{\prime}$ contributions to the potential as in [2]. This provides a class of models without the need for an antibrane in a warped throat and may facilitate more explicit model-building analyses, since computations are easier in symmetric toroidal orientifolds of the sort we studied. We also regard our success in finding explicit models in the toroidal case suggestive of their existence in the richer context of generic Calabi-Yau manifolds. The uniformly non-negative mass-squares we encountered in the symmetry breaking directions were a pleasant surprise, and it would be nice to find a simple analytical explanation for that result. The hierarchy of mass scales appearing in our results is also intriguing and may have interesting applications.

\section{Acknowledgements}

We thank Wu-yen Chuang for early collaboration and P. Candelas, D. Freed, S. Kachru, X. Liu, L. McAllister, and M. Schulz for many helpful discussions. We are supported in part by the DOE under contract DE-AC03-76SF00515 and by the NSF under contract 9870115 .

\section{Appendix A. Data}

All solutions have the same choices for 12 of the fluxes:

$$
\begin{aligned}
\left(b_{0}, b_{11}, b_{22}, b_{33}\right) & =(4,0,0,0) \\
\left(c^{0}, c^{11}, c^{22}, c^{33}\right) & =(4,-2,-2,-2) \\
\left(d_{0}, d_{11}, d_{22}, d_{33}\right) & =(-4,-2,-2,-2) .
\end{aligned}
$$

The locations and first derivatives of all vacua were calculated to at least 12 significant figures, though only a few are given here, and all calculated values claimed to be zero were smaller than $10^{-12}$ and of the same order as other calculational artifacts. In the first table, the integer under D3 is the number of D3-branes required to satisfy (2.10).

\begin{tabular}{|c|cccc|c|c|}
\hline Vacuum & $a^{0}$ & $a^{11}$ & $a^{22}$ & $a^{33}$ & D3s & $V \cdot\left(\alpha^{\prime}\right)^{2}(\rho-\bar{\rho})^{3}$ \\
\hline $\mathrm{A}$ & 4 & 2 & 0 & -2 & 0 & $7.424 \times 10^{-6}$ \\
\hline $\mathrm{B}$ & 4 & 0 & -2 & -2 & 4 & $1.029 \times 10^{-4}$ \\
\hline $\mathrm{C}$ & 4 & 0 & 0 & -2 & 2 & $3.609 \times 10^{-5}$ \\
\hline $\mathrm{D}$ & 4 & -2 & -2 & 2 & 2 & $4.233 \times 10^{-5}$ \\
\hline
\end{tabular}




\begin{tabular}{|c|cccc|}
\hline Vac. & $\tau^{11}$ & $\tau^{22}$ & $\tau^{33}$ & $\phi$ \\
\hline $\mathrm{A}$ & $2.653-1.851 \mathrm{i}$ & $2.032-0.4752 \mathrm{i}$ & $0.2534-0.4578 \mathrm{i}$ & $0.9022+0.8783 \mathrm{i}$ \\
\hline $\mathrm{B}$ & $1.477-1.427 \mathrm{i}$ & $0.6650-0.5250 \mathrm{i}$ & $0.6650-0.5250 \mathrm{i}$ & $1.177+0.4567 \mathrm{i}$ \\
\hline $\mathrm{C}$ & $1.523-0.7046 \mathrm{i}$ & $1.523-0.7046 \mathrm{i}$ & $0.4489-0.4800 \mathrm{i}$ & $1.161+0.5285 \mathrm{i}$ \\
\hline $\mathrm{D}$ & $0.5846-0.4487 \mathrm{i}$ & $0.5846-0.4487 \mathrm{i}$ & $1.225-2.835 \mathrm{i}$ & $0.8017+1.108 \mathrm{i}$ \\
\hline
\end{tabular}

The following tables show the eigenvalues of the mass matrix at the minima, in the 23 real directions of all metric moduli and the axion-dilaton.

\begin{tabular}{|c|ccccc|}
\hline Vac. & \multicolumn{5}{|c|}{ Mass matrix eigenvalues of $V \cdot\left(\alpha^{\prime}\right)^{2}(\rho-\bar{\rho})^{3}$} \\
\hline A & 0.4404 & 0.09416 & 0.06495 & 0.03706 & 0.004580 \\
& 0.002429 & 0.001306 & 0.001286 & $7.215 \times 10^{-4}$ & $6.756 \times 10^{-4}$ \\
& $6.192 \times 10^{-4}$ & $3.862 \times 10^{-4}$ & $3.652 \times 10^{-4}$ & $3.635 \times 10^{-4}$ & $2.996 \times 10^{-4}$ \\
& $1.831 \times 10^{-4}$ & $1.597 \times 10^{-4}$ & $1.487 \times 10^{-4}$ & $9.190 \times 10^{-6}$ & $1.802 \times 10^{-7}$ \\
& 0 & 0 & 0 & & \\
\hline B & 0.02050 & 0.01523 & 0.01523 & 0.008724 & 0.008334 \\
& 0.007821 & 0.007525 & 0.0023505 & 0.001444 & $9.480 \times 10^{-4}$ \\
& $9.480 \times 10^{-4}$ & $2.846 \times 10^{-4}$ & $1.781 \times 10^{-4}$ & $1.430 \times 10^{-4}$ & $1.074 \times 10^{-4}$ \\
& $1.074 \times 10^{-4}$ & $7.303 \times 10^{-5}$ & $2.791 \times 10^{-5}$ & $2.791 \times 10^{-5}$ & 0 \\
& 0 & 0 & 0 & & 0 \\
\hline
\end{tabular}

\begin{tabular}{|c|ccccc|}
\hline Vac. & \multicolumn{5}{|c|}{ Mass matrix eigenvalues of $V \cdot\left(\alpha^{\prime}\right)^{2}(\rho-\bar{\rho})^{3}$} \\
\hline $\mathrm{C}$ & 0.05010 & 0.02131 & 0.02131 & 0.01151 & 0.002869 \\
& 0.001775 & 0.001629 & 0.001401 & 0.001401 & 0.001258 \\
& $7.722 \times 10^{-4}$ & $6.701 \times 10^{-4}$ & $3.245 \times 10^{-4}$ & $2.281 \times 10^{-4}$ & $2.281 \times 10^{-4}$ \\
& $1.679 \times 10^{-4}$ & $1.080 \times 10^{-4}$ & $1.080 \times 10^{-4}$ & $3.813 \times 10^{-5}$ & 0 \\
& 0 & 0 & 0 & & \\
\hline $\mathrm{D}$ & 0.07233 & 0.07233 & 0.07027 & 0.01616 & 0.01279 \\
& 0.008878 & 0.007767 & 0.005338 & 0.002045 & 0.001176 \\
& $8.341 \times 10^{-4}$ & $8.341 \times 10^{-4}$ & $3.082 \times 10^{-4}$ & $3.082 \times 10^{-4}$ & $1.971 \times 10^{-4}$ \\
& $1.098 \times 10^{-4}$ & $9.928 \times 10^{-5}$ & $3.293 \times 10^{-5}$ & $3.293 \times 10^{-5}$ & 0 \\
& 0 & 0 & 0 & & \\
\hline
\end{tabular}




\section{References}

[1] A. Maloney, E. Silverstein and A. Strominger, "De Sitter space in noncritical string theory," arXiv:hep-th/0205316; E. Silverstein, "(A)dS backgrounds from asymmetric orientifolds," arXiv:hep-th/0106209.

[2] S. Kachru, R. Kallosh, A. Linde and S. P. Trivedi, "De Sitter vacua in string theory," Phys. Rev. D 68,046005 (2003) arXiv:hep-th/0301240].

[3] B. S. Acharya, "A moduli fixing mechanism in M theory," arXiv:hep-th/0212294.

[4] E. Silverstein, "AdS and dS entropy from string junctions," arXiv:hep-th/0308175.

[5] M. Fabinger and E. Silverstein, "D-Sitter space: Causal structure, thermodynamics, and entropy," arXiv:hep-th/0304220.

[6] R. Bousso and J. Polchinski, JHEP 0006, 006 (2000) arXiv:hep-th/0004134.

[7] L. Susskind, "The anthropic landscape of string theory," arXiv:hep-th/0302219.

[8] S. Ashok and M. R. Douglas, "Counting flux vacua," arXiv:hep-th/0307049.

[9] S. Kachru, J. Pearson and H. Verlinde, "Brane/flux annihilation and the string dual of a non-supersymmetric field JHEP 0206, 021 (2002) arXiv:hep-th/0112197.

[10] C. P. Burgess, R. Kallosh and F. Quevedo, "de Sitter string vacua from supersymmetric D-terms," JHEP 0310, 056 (2003) arXiv:hep-th/0309187.

[11] S. B. Giddings, S. Kachru and J. Polchinski," 'Hierarchies from fluxes in string compactifications," Phys. Rev. D 66, 106006 (2002) arXiv:hep-th/0105097.

[12] S. Kachru, M. B. Schulz and S. Trivedi, "Moduli stabilization from fluxes in a simple IIB orientifold," JHEP 0310, 007 (2003) arXiv:hep-th/0201028.

[13] A. R. Frey and J. Polchinski, "N = 3 warped compactifications," Phys. Rev. D 65, 126009 (2002) arXiv:hep-th/0201029.

[14] P. K. Tripathy and S. P. Trivedi, "Compactification with flux on K3 and tori," JHEP 0303, 028 (2003) arXiv:hep-th/0301139.

[15] J. D. Brown and C. Teitelboim, "Neutralization Of The Cosmological Constant By Membrane Creation," Nucl. Phys. B 297, 787 (1988).

[16] S. Kachru, X. Liu, M. B. Schulz and S. P. Trivedi, "Supersymmetry changing bubbles in string theory," JHEP 0305, 014 (2003) arXiv:hep-th/0205108.

[17] K. Dasgupta, G. Rajesh and S. Sethi, "M theory, orientifolds and G-flux," JHEP 9908, 023 (1999) arXiv:hep-th/9908088.

[18] L. Kofman, A. Linde, X. Liu, A. Maloney, L. McAllister, and E. Silverstein, "Moduli Trapping from Particle Production", to appear.

[19] M. Dine, "Towards a Solution of the Moduli Problems of String Cosmology," Phys.Lett. B482 (2000) 213, hep-th/0002047;

M. Dine, Y. Nir, and Y. Shadmi, "Enhanced Symmetries and the Ground State of String Theory," Phys.Lett. B438 (1998) 61, hep-th/9806124. 
[20] S. Gukov, C. Vafa and E. Witten, "CFTs from Calabi-Yau Fourfolds," Nucl. Phys. B584, 69 (2000) arXiv:hep-th/9906070

[21] T. R. Taylor and C. Vafa, "RR flux on Calabi-Yau and partial supersymmetry breaking," Phys. Lett. B 474, 130 (2000) arXiv:hep-th/9912152.

[22] P. Mayr, "On supersymmetry breaking in string theory and its realization in brane Nucl. Phys. B 593, 99 (2001) arXiv:hep-th/0003198.

[23] L. Andrianopoli, S. Ferrara and M. Trigiante, "Fluxes, supersymmetry breaking and gauged supergravity," arXiv:hep-th/0307139.

[24] J. Polchinski, "String Theory. Vol. 2: Superstring Theory And Beyond," Cambridge, UK: Univ. Pr. (1998) 531 p.

[25] S. Wolfram, "The Mathematic Book," Wolfram Media/Cambridge Univ. Pr., 1999 